\title{
Purified Wheat Gliadin Proteins as Immunoglobulin E Binding Factors in Wheat Mediated Allergies
}

\author{
Jacek Waga ${ }^{1}$, Krystyna Obtułowicz ${ }^{2}$, Jerzy Zientarski ${ }^{1}$, Ewa Czarnobilska ${ }^{2}$, Andrzej Skoczowski ${ }^{3}$ \\ ${ }^{1}$ Cereals Department, Plant Breeding and Acclimatization Institute, Krakow, Poland; ${ }^{2}$ Department of Clinical and Environmental \\ Allergology, Collegium Medicum Jagiellonian University, Krakow, Poland; ${ }^{3}$ Institute of Biology, Pedagogical University, Krakow, \\ Poland. \\ Email: zhwaga@cyf-kr.edu.pl
}

Received June $6^{\text {th }}, 2011$; revised August $15^{\text {th }}, 2011$; accepted August $22^{\text {nd }}, 2011$.

\begin{abstract}
Some wheat gliadin proteins are strong allergens that may cause various symptoms of food allergies and baker's asthma. The most immunoreactive $\omega-5$ gliadin fractions are the main allergens in wheat dependent exercise induced anaphylaxis (WDEIA). While the allergenicity of $\omega-5$ is quite well understood, knowledge about $\alpha, \beta, \gamma$ and $\omega-1.2$ gliadins is much more scanty. This study examines allergenic properties of other fractions as compared to $\omega-5$. Gliadins were extracted from flour of winter wheat (Triticum aestivum L.) cultivar Ostka strzelecka. Purified samples representing proteins belonging to $\alpha, \beta, \gamma, \omega-1.2$ and $\omega-5$ classes were isolated using preparative gel electrophoresis. Immunoreactivity and allergenic properties of these proteins were analyzed by ELISA using sera from allergic patients with elevated sIgE (>2KU/L), and by skin prick test (SPT). ELISA showed that $\omega-5$ and $\omega-1.2$ differed considerabely from $\alpha$-, $\beta$ - and $\gamma$-gliadins in respect of immunoreactivity. Responses of both $\omega$-gliadins were almost twice as high as for other fractions. Significant differences were also observed among individual $\omega$-gliadin fractions as evidenced by ANOVA. SPT showed that patient with symptoms of baker's asthma and WDEIA had a positive results to all gliadins tested. Another patient with baker's asthma (but not WDEIA) reacted positively only to $\omega-5$ gliadins. In two patients with skin allergy SPT were negative with all analyzed proteins. Results show $\omega-1.2$ gliadins to be almost as immunorective as $\omega-5$. The $\alpha-, \beta$ - and $\gamma$-gliadins also recognize specific IgE antibodies, but their binding capacity is only about half that of $\omega$-fractions. This kind of immunoreactivity could still be important since a cumulative effect of individual fractions may intensify disease symptoms in allergic patients.
\end{abstract}

Keywords: Allergies, Gliadins, Immunoreactivity, Preparative A-PAGE, Wheat

\section{Introduction}

Gliadin proteins are functional components of most wheatfood products. However, for people with celiac disease or specific wheat food allergies, they may be toxic or allergenic factors. Since gliadins (as gluten proteins) are important in human nutrition throughout the world, their celiac toxicity and allergenicity are serious problems for both medical and nutrition sciences.

Gliadins are unique, highly atypical and the most complex and heterogeneous protein group in the plant kingdom. They are composed of hundreds of monomeric proteins, rich (like all prolamines) in proline (ca. 14\%) and glutamine (ca. 40\%) [1,2]. The great polymorphism of gliadin proteins is determined genetically. Gliadins are coded by six polygenic loci on chromosomes 1 and 6 of the A, B, and $\mathrm{D}$ genomes of hexaploid wheat (Triticum aestivum $\mathrm{L}$.) [3]. Each locus contain from several to tens of individual, closely linked genes. Many individual gliadin genes have been duplicated throughout evolution. Their structures subsequently changed by point mutation, unequal crossing-over, gene silencing, translocation, and deletion of DNA fragments. Consequently, multiple alleles for each locus arose during evolution of hexaploid wheat. Each allele codes for specific groups of polypeptides defined as gliadin protein blocks [4]. Genetic studies show proteins of each block to be expressed and inherited together. Thus, individual wheat genotype contains tens of distinct monomeric gliadins, differing in sequence, chemical properties, and physicochemical structure. Moreover, gliadin polypeptides compose a number of combinations among different wheat genotypes. Hence wheat species, cultivars and breeding lines vary greatly in gliadin composition. This heterogeneity, as well as inter-varietal differences, have been shown by acid polyacrylamide gel 
electrophoresis (A-PAGE), high performance capillary electrophoresis (HPCE) and reversed phase high performance liquid chromatography (RP-HPLC) [5-7].

Gliadins also have unique biochemical structures, containing both repeating and non-repeating sequences. For example, $\alpha$-gliadins are rich in short repeated sequences localized near the $\mathrm{N}$-terminal regions, while their C-terminal regions are unique, rich in glutamine and contain six cysteine residues that form intra-molecular disulphide bonds [8-10]. In contrast, $\omega$-gliadins have no cysteine residues and consist mainly of short, repeated sequences, with the tetrapeptides QPQQ and PQQP being most frequent (23 and 30 repetitions respectively) [11]. Repeated sequences often form epitopes that bind IgE antibodies present in elevated amounts in sera of patients sensitive to gluten. Multiple epitopes may amplify formation of IgE-gliadin complexes and-in consequence-protein immunoreactive properties.

It seems obvious that such great differentiation can significantly influence allergenicity of individual proteins within the protein complex. The allergenic properties of $\omega-5$ gliadins are rather precisely known. These are the main allergens in wheat-dependent exercise induced anaphylaxis (WDEIA), the gluten protein allergy most dangerous for human life $[12,13]$. However, there have been far fewer studies of $\alpha, \beta, \gamma$ and $\omega-1.2$ gliadins. These proteins may also significantly impact allergenicity, since all gliadins share many related sequence characteristics and epitopes. Thus, the allergenicity of the entire protein complex may result from additive effects of its individual components. In addition, each of five $\alpha, \beta, \gamma, \omega-5$ and $\omega-1.2$ gliadin classes is composed of several proteins, appearing as individual bands upon A-PAGE. No detailed studies have been performed to identify the most allergenic fractions among $\alpha, \beta, \gamma, \omega-5$ and $\omega-1.2$ groups. It seems probable that individual gliadin fractions may also differ from each other in allergenicity; this may be important in allergology to identify major allergens immunologically active in various allergies, and in plant breeding for development of wheat genotypes having decreased allergenic properties.

Recent studies have considerably advanced the understanding of cereal proteins immunology, but knowledge of wheat gliadins as allergens is still incomplete [14]. Important aspects concerning protein structure to allergenicity remain unexplained. One such aspect is the relation of allergenic properties to polymorphism. Studies on allergenicity of gluten proteins are multidisciplinary, with medical and biochemical sciences playing major roles in this field. Medical sciences are usually directed to characterize symptoms of allergic diseases among large populations of subjects sensitized to predicted allergens. Biochemical research has attempted to identify the most immunoreactive peptides and epitopes among allergenic proteins. The aim of the present study-more biochemical than medical in approach-is to isolate and characterize immunoreactive properties of individual, purified gliadin proteins, belonging to $\alpha, \beta, \gamma, \omega-5$ and $\omega-1.2$ groups. Special attention was paid to the allergenicity of $\alpha, \beta, \gamma$, and $\omega-1.2$ as compared to $\omega-5$ gliadin fractions.

\section{Material and Methods}

\subsection{Plant Material}

The winter wheat cultivar Ostka strzelecka, from the collection of Plant Breeding and Acclimatization Institute, Cereals Department, Krakow (PBAI), harvested in 2008, was used in this study. Kernels were milled on a Brabender Quadrumat Senior mill (Brabender $\mathrm{GmbH}$, Germany) having sieves with mesh diameters from 150 $\mu \mathrm{m}$ to $500 \mu \mathrm{m}$. The resulting flour was used for gliadin extraction.

\subsection{Purification of Gliadins by Preparative A-PAGE}

Gliadins were extracted from flour with 70\% EtOH (1:10 $\mathrm{v} / \mathrm{w})$ and gentle shaking on a Grant-bio PTR-60 rotator at $15 \mathrm{rpm} / \mathrm{min}$ overnight at room temperature. After centrifugation $(12,500 \mathrm{x} \cdot \mathrm{g}, 10 \mathrm{~min})$, extracts were introduced into aluminium lactate buffer $(\mathrm{pH}=3.1)$ saturated with sucrose in the proportion $1: 1$. An aliquot $(2.7 \mathrm{~mL})$ of this was applied to the surface of a polyacrylamide gel column $(\mathrm{T}=8 \%, \mathrm{C}=0.29 \%)$ in a Model 491 PrepCell electrophoretic apparatus (BioRad, USA) and gliadins were fractionated by A-PAGE [15]. The total amount of gliadin proteins in extracts applied to the column was approximately $10 \mathrm{mg}$. The gel column was $10 \mathrm{~cm}$ high and $37 \mathrm{~mm}$ wide. Separations were done at constant power $(11 \mathrm{~W})$; maximum voltage was set at $320 \mathrm{~V}$, and current varied during electrophoresis from 41 to $19 \mathrm{~mA}$. At the bottom of the gel column an elution chamber equipped with frits (BioRad patent 4.877.510) was installed. The elution chamber was connected via a silica connector with a chamber containing eluent solution $(0.01 \mathrm{M}$ acetic acid). Fractions leaving the column were collected beginning two hours after the start of electrophoresis. Separated proteins were transferred from the elution chamber to a fraction collector (Model 2110, BioRad, USA) with a peristaltic pump. It was thus possible to collect 80 aliquots from a protein mixture during 12 hours.

Aliquots from preparative separations were tested twice by analytical A-PAGE [16]. The first separation, to identify gliadin fractions, was done in a Desaphor Electrophoretic Chamber (Desaga GmbH, Germany). $200 \mu \mathrm{L}$ 
of each aliquot was mixed with $90 \mu \mathrm{L}$ of aluminium lactate buffer $(\mathrm{pH}=3.1)$ saturated with sucrose, and introduced into electrophoretic wells in the polyacrylamide gel. Proteins were separated for four hours at constant voltage $(500 \mathrm{~V})$. Based on the obtained results, aliquots containing identical protein bands were combined and concentrated to $1.5 \mathrm{~mL}$ with a CentriVap Concentrator (Labconco, USA). Resulting fractions were then again electrophoresed by A-PAGE using Mini Protean 3 (BioRad, USA) to check their purity. The second separation was done for 130 minutes at constant voltage $(155 \mathrm{~V})$; current varied during electrophoresis from 12 to $9 \mathrm{~mA}$. The protein content of each fraction was determined by the Bradford method using Bio-Rad Protein Assay Kit I (Bio-Rad, USA). Purified protein samples for further analysis were then lyophilized and stored at $4^{\circ} \mathrm{C}$. Using these methods, it was possible to obtain $0.3 \mathrm{mg}$ to $1.0 \mathrm{mg}$ of individual fractions.

\subsection{Patients}

Four patients (two men and two women, ages $25-55$ ) with increased $\operatorname{sigE}$ serum level to gluten $(>2 \mathrm{KU} / \mathrm{L}$, UniCap Pharmacia, Sweden), treated in Outpatients Department of Allergology in University Hospital in Krakow, were included in this study (Table 2). Two of these subjects were bakers with baker's asthma, developed at work from exposure to wheat dust. One of these also developed anaphylaxis after consuming whole grain bread (gluten ingestion) after physical exercise [WDEIA]. The serum level of sIgE to gluten in both patients was high $(11.4 \mathrm{KU} / \mathrm{L}$ and $12.3 \mathrm{KU} / \mathrm{L}$, compared to the normal value of $<0.35 \mathrm{KU} / \mathrm{L})$. The third patient, exhibited atopic dermatitis and atopic eczema, was more severe after skin contact with wheat flour. Patient four also exhibited skin symptoms (urticaria) after skin contact with wheat flour. The serum level of sIgE to gluten in third and fourth patients was elevated but much lower than in patients with bronchial asthma $(2.12 \mathrm{KU} / \mathrm{L}$ and $3.36 \mathrm{KU} / \mathrm{L})$.

\subsection{ELISA}

Binding capacity of purified gliadin fractions by $\operatorname{IgE}$ antigliadin antibodies from allergic patients sera was analyzed by immunoenzymatic indirect ELISA. Proteins were diluted in carbonate buffer $(0.05 \mathrm{~mol} / \mathrm{L}, \mathrm{pH}=9.6)$ and microtiter wells were coated with $100 \mu \mathrm{L}$ of analyzed solutions overnight at $4^{\circ} \mathrm{C}$. Protein concentration of all samples was adjusted to $100 \mu \mathrm{g} / \mathrm{mL}$. Each sample was analyzed in four replicates. In blank samples $1 \%$ foetal calf serum (FCS) diluted in phosphate-buffered saline with addition of $1 \%$ Tween (PBST), was introduced into the wells instead of gliadin samples. On the next day all wells of the microtiter plates were blocked with $1 \%$ FCS at room temperature for two hours. After removing the blocker, pooled sera of all patients, diluted 1:5 in PBST, were introduced to microtiter wells. Plates were incubated for two hours at $37^{\circ} \mathrm{C}$. After incubation the sera were removed and $100 \mu \mathrm{L}$ of the secondary antibody solution ( $\xi$-chain of $\operatorname{IgE}$ antihuman antibodies conjugated with horseradish peroxidase, Sigma Co., USA) diluted 1:1000 in PBST was added to each well and incubated again for two hours at $37^{\circ} \mathrm{C}$. After each stage of ELISA, wells were rinsed three times with PBST. The substrate for horseradish peroxidase was O-Phenylenediamine prepared according to manufacturer's instruction (Sigma Co., USA). Reaction with substrate was carried out for 20 minutes in darkness, and stopped by adding $100 \mu \mathrm{L}$ of $3 \mathrm{M} \mathrm{H}_{2} \mathrm{SO}_{4}$ to each well. Optical density (OD) of coloured products of the immunoenzymatic reaction was estimated with an ELISA plate reader (Opsys MR, Thermolab System, USA) at $490 \mathrm{~nm}$. Average values from four repetitions, multiplied by 1000 , were accepted as indicators of protein immunoreactivity. Statistical significance of differences among average values of the ODx1000 indicators was estimated by one dimensional ANOVA. Least significant difference (LSD) intervals at $P=95 \%$ were estimated by the Tukey test. ANOVA and Tukey tests were performed using the program Statigraphics Plus 1.4.

\subsection{Skin Prick Tests}

In all patients studied, skin prick tests (SPT) were performed according to standard method [17] with prepared gliadin fractions in concentration $200 \mathrm{ug} / \mathrm{ml}$, as well as with positive (1\% histamine) and negative (Allergopharma, Germany) control. The skin reactions were evaluated after 15 minutes: wheals $\geq 3 \mathrm{~mm}$ diameter were judged positive at negative control $-0 \mathrm{~mm}$ and positive control-3 $\mathrm{mm}$ or more.

\section{Results}

A-PAGE of gliadin proteins from winter wheat cultivar Ostka strzelecka reveals 25 bands (Figure 1).

The $\omega-5$ and $\omega-1.2$ groups contain three and five bands respectively. All $\omega$-gliadins are composed on single bands, well-marked and clearly separated from each other. The remaining groups of $\alpha$-, $\beta$ - and $\gamma$-gliadins contain seven, six and four bands respectively. Some bands are much thicker and closer than in $\omega$ fractions. That is because the $\alpha, \beta$ and $\gamma$ zones of A-PAGE electrophoregrams commonly superimpose many polypeptides having similar or identical mobilities, making identification and purification a serious methodological problem.

Preparative A-PAGE fractionated gliadins from Ostka Strzelecka into 80 aliquots, which were than analyzed by analytical A-PAGE. Aliquots with identical protein 
compositions were combined, concentrated and lyophilized. Twelve purified protein samples were thus obtained for further studies (Figure 2).

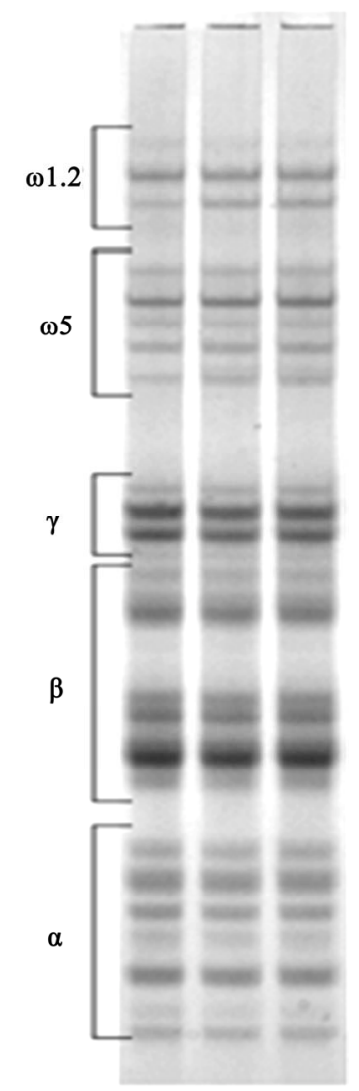

Figure 1. A-PAGE electrophoregrams of gliadin patterns of winter wheat cultivar Ostka strzelecka. $\alpha, \beta, \gamma, \omega-5$ and $\omega-1.2$ (indicated on the left hand side of the electrophoregram): groups of gliadin proteins varied in regard of electrophoretic mobility.

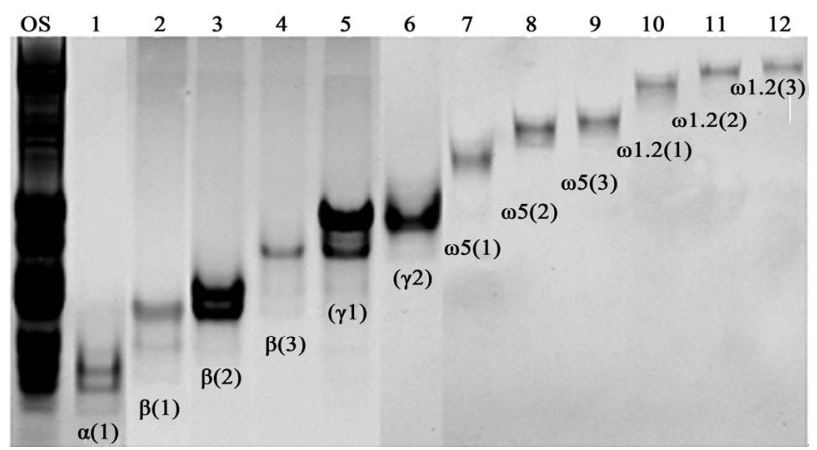

Figure 2. A-PAGE electrophoregram of purified gliadin fractions obtained from winter wheat cultivar Ostka strzelecka. OS-pattern of gliadin proteins complex specific for cultivar Ostka strzelecka. 1, 2, $3 \cdots 12$ (on the top of the picture): the number of the runs. $\alpha[1], \beta[1], \beta[2] \cdots \omega-1.2[3]$ (under the protein bands): symbols of individual gliadin protein fractions.
One of these samples contained $\alpha$-gliadins, three contained $\beta$-, two $\gamma$-, three $\omega-5$ and three contained $\omega-1.2$ gliadin fractions. The first sample $(\alpha[1])$ was composed on two $\alpha$-gliadin bands. The next 3 samples comprised $\beta$-gliadins (2-4: $\beta[1], \beta[2]$ and $\beta[3])$. Two of these $(\beta[1]$ and $\beta[3])$ were composed on single bands whereas the third $(\beta[2])$ combined two bands: $\beta[1]$ and $\beta[3]$. Samples 5 and 6 contained $\gamma$-gliadins. Sample $5(\gamma[1])$ has an intense band with several superimposed proteins-products of genes localized on different chromosomes, plus some of the $\beta[3]$ band; sample 6 appears to contain only one $\gamma$-gliadin $(\gamma[2])$. Two subclasses of the $\omega$-gliadins $(\omega-1.2$ and $\omega-5)$ were examined separately. For each subclass three samples $(7,8,9$ and 10,11, 12) were obtained; each sample contained one major band ( $\omega-5[1], \omega-5[2], \omega-5[3]$ and $\omega-1.2[1], \omega-1.2[2], \omega-1.2[3])$.

Indirect ELISA showed large differences among immunoreactive properties of twelve purified gliadin fractions, as revealed by binding capacity to serum $\operatorname{IgE}$ antibodies of patients allergic to gluten. ANOVA confirmed these differences to be statistically significant $(P<0.001)$ (Table 1).

For all average OD values, least significant difference (LSD, $P=95 \%$ ) and high and low limits of average values were calculated by the Tukey test. Observed OD values for subclasses $\omega-1.2$ and $\omega-5$ were almost twice as high as for other protein fractions. The highest OD values were for fractions $\omega-5$ [2] and $\omega-1.2[3]$, and the lowest for $\gamma[1]$ (Figure 3).

OD variability ranges of most $\alpha$-, $\beta$ - and $\gamma$-proteins did not differ significantly however, $\gamma(1)$ was significantly lower in OD value than $\alpha(1), \beta(1)$ and $\beta(3)$ fractions. Many significant differences occurred among $\omega$-gliadin fractions. The average OD value for $\omega-1.2[1]$ was significantly lower than for $\omega-5[1], \omega-5[2], \omega-1.2[2]$ and $\omega-1.2[3]$. Similarly, the value for $\omega-5[3]$ was significantly lower than for $\omega-5[1], \omega-5[2]$ and $\omega-1.2[3]$, while the value for $\omega-1.2[2]$ was significantly lower than for $\omega-5$ [2] and $\omega-1.2[3]$.

Table 1. One way analysis of variance for average OD values obtained by ELISA of purified gliadin fractions from winter wheat Ostka strzelecka using pooled sera of four patients allergic to gluten.

\begin{tabular}{ccccc}
\hline Source of variation & $\boldsymbol{S S}$ & $\boldsymbol{d f}$ & Variance & $\boldsymbol{F}$ \\
\hline Overall & 515218 & 35 & & \\
$\begin{array}{c}\text { Between groups } \\
\text { (proteins) }\end{array}$ & 496219 & 11 & 45111 & $56,96^{* * *}$ \\
$\begin{array}{c}\text { Among groups } \\
\text { (error) }\end{array}$ & 18999 & 24 & 792 & \\
\hline
\end{tabular}

$S S$-sum of squares; $d f$-degrees of freedom; $* * *$ - significant at $P<0.001$. 
Allergenic properties of five chosen purified gliadins $(\alpha[1], \beta[1], \gamma[2], \omega-5[2]$ and $\omega-1.2[3])$, were evaluated by skin tests (SPT) on patients who also donated serum for ELISA. Patient 1, with symptoms of baker's asthma and WDEIA and the highest specific IgE level, had positive results for all fractions tested (Table 2).

Patient 2, having symptoms of baker's asthma but not
WDEIA, and having a specific IgE level similar to patient 1 , reacted positively only to gliadin $\omega-5[2]$; this fraction was also most immunoreactive by ELISA. Patients 3 and 4 (with skin symptoms and low levels of specific IgE antibodies) showed SPT wheals slightly larger than the negative control, but this could not be evaluated as a positive reaction.

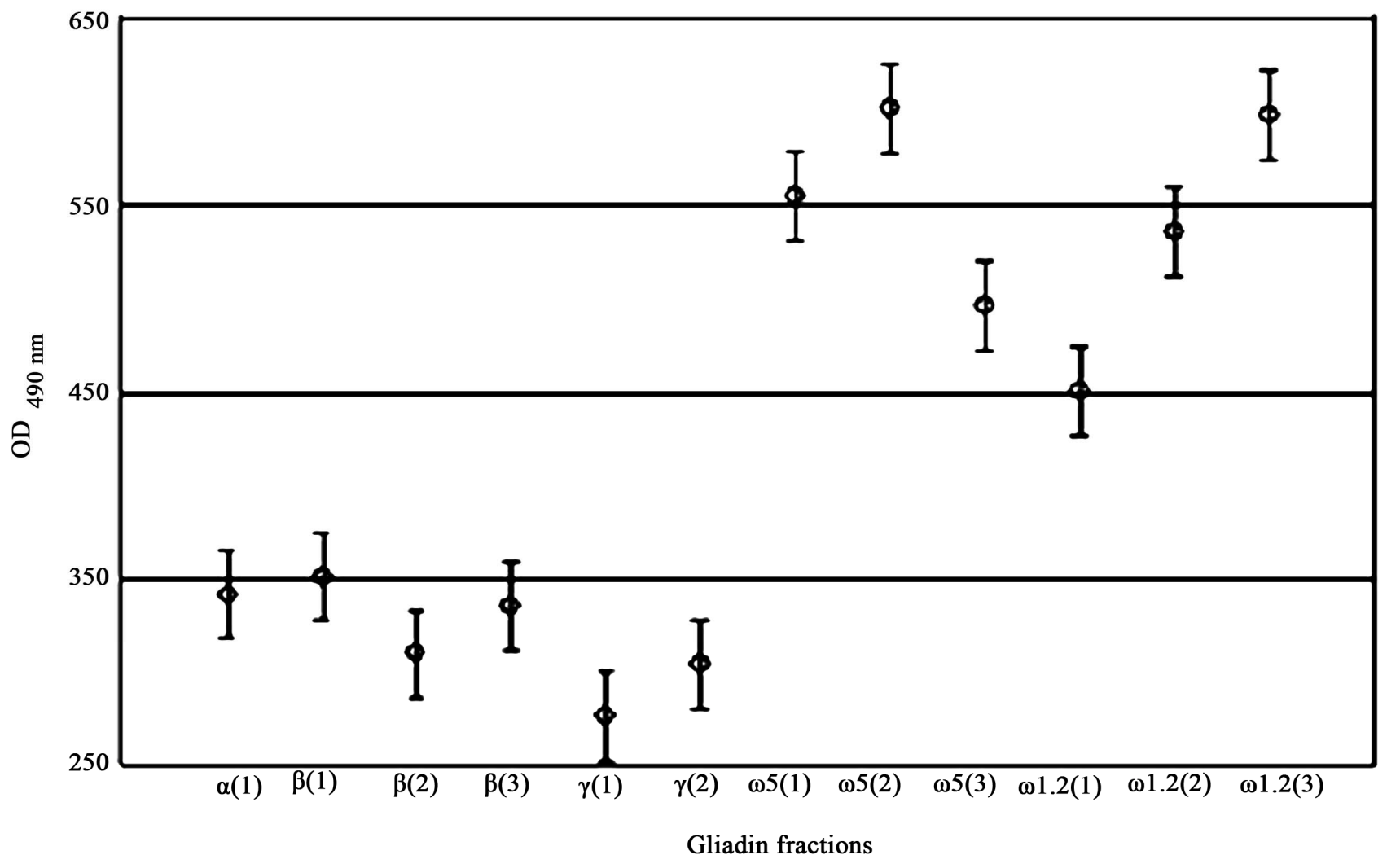

Figure 3. Average optical densities (OD values) obtained by ELISA, and LSD intervals $(P=95 \%)$ estimated by Tukey test for purified gliadin fractions of cultivar Ostka strzelecka in reaction with human sera from patients allergic to gluten. $\alpha[1], \beta[1]$, $\beta[3], \cdots, \omega-1.2[3]$ (on the $X$-axis): Symbols of purified gliadin fractions (compatible with Figure 2). 250, 350, $\cdots, 650$ (on the Y-axis): absorbance (OD) values of ELISA multiplied by 1000 used as gliadin's immunoreactivity indicators (the higher OD value the highest immunoreactivity of the gliadin fraction).

Table 2. Results of skin prick tests performed for patients allergic to gluten (sIgE- $\mathrm{f}_{76}>\mathbf{2 . 0} \mathrm{IU} / \mathrm{mL}$ ) using individual gliadin fractions as antigens.

\begin{tabular}{|c|c|c|c|c|c|c|c|c|c|}
\hline \multirow{2}{*}{ Patients } & \multirow{2}{*}{ sIgE (IU/L) } & \multirow{2}{*}{ Symptoms of disease } & \multicolumn{7}{|c|}{ Wheal diameter (mm) } \\
\hline & & & $\alpha$ & $\beta$ & $\gamma$ & $\omega 5$ & $\omega 1.2$ & $\mathrm{NC}$ & $\mathrm{PC}$ \\
\hline 1 & 12.3 & Baker asthma, WDEIA & $>3$ & $>3$ & $>3$ & $>3$ & $>3$ & 0 & $>3$ \\
\hline 2 & 11.4 & Baker asthma & 2 & 2 & 2 & $>2$ & 2 & 0 & $>3$ \\
\hline 3 & 3.36 & Skin symptoms, AD & 1.5 & 1.5 & 1.5 & 1.5 & 1.5 & 0 & $>3$ \\
\hline 4 & 2.12 & Skin symptoms, URT & 1.5 & 1.5 & 1.5 & 1.5 & 1.5 & 0 & $>3$ \\
\hline
\end{tabular}

$\mathrm{NC}$-Negative control $(0.9 \% \mathrm{NaCl}$ solution); PC—positive control (1\% histamine); AD—atopic dermatitis; URT—urticaria. 


\section{Discussion}

In the present studies we aimed to obtain information about immunoreactive properties of isolated and purified gliadin fractions. Therefore we observed the effects of immunological reaction between individual proteins and IgE antibodies using pooled sera of four patients showing different symptoms of gluten protein intolerance for ELISA. Immunoreactive properties of characterized gliadins were then verified using skin prick test on patients who also donated sera for this study. In spite of the fact that patients 1 and 2 (in accord with Table 2) showed significantly higher sIgE levels than patients 3 and 4, each having antibodies which increased the total amount of sIgE in the polled sera. However, antibodies present in sera of patients showing various symptoms of wheat gluten protein allergies may slightly differ each other in respect of amino acid sequences in hypervariable regions and-in consequence-may bind to different epitopes of gliadin polypeptides. Hence, we expected pooled sera to show broad immunological activity, and antibodies of each patient contribute to immunoreactivity of analyzed proteins as measured by ELISA. A similar strategy has been used in other studies on wheat protein allergenicity. Watanabe et al. [18] identified allergenic peptides in gluten using serum of one patient, while Tanabe et al. [19] used pooled sera of four patients to show that peptide QQQPP, common in gluten proteins, is a strong $\operatorname{IgE}$ binding epitope.

The complexity of gliadin proteins complicates efforts to recognize and characterize the biochemical bases of gliadin allergenicity. We are convinced that studies of individual, native gliadin fractions can provide more objective and valuable information about their immunoreactivity than can analysis of the whole gliadin complex or even separate $\alpha, \beta, \gamma$ and $\omega$ classes. We therefore investigated allergenic properties of highly purified proteins isolated by preparative A-PAGE. This method is useful for analysis of gliadin's immunoreactive properties, as confirmed in our previous studies and by others $[15,20]$. It is thus possible to obtain purified fractions that have the immunological characteristics for native proteins; preparative A-PAGE permits isolation of proteins in amounts sufficient for immunoassays (ELISA and SPT), as described in this paper.

Results in Figure 3 reveal relationships of both gliadin classes $(\alpha, \beta, \gamma, \omega-5$ and $\omega-1.2)$ and individual gliadin fractions to immunoreactivity. Observed differences in immunoreactive properties among gliadin classes permit dividing the analyzed proteins into two groups: the $\omega$-proteins ( $\omega-5$ and $\omega-1.2)$ and the remaining $\alpha, \beta$ and $\gamma$ proteins. Immunoreactivity of the $\omega$-gliadins was much higher than that of the other gliadins. Our ELISA results confirm the highest IgE binding capacity for the class $\omega-5$. These results were analogous to those of others $[12,13,21]$. We found, however, that $\omega-1.2$ gliadins have immunoreactivity similar to or even greater than $\omega-5$. The $\alpha, \beta$ and $\gamma$ gliadins may also bind specific IgE antibodies of patients allergic to gluten. Their immunological activity is much lower than that of both $\omega$ subclasses, but could be important especially, if there is an additive effect of individual proteins on disease development. This assumption is partly strengthened by the results of proteomic studies of Akagawa et al. [22] who showed that $\gamma$-gliadins may be important wheat flour allergens using pooled sera from seven patients allergic to gluten.

We must emphasize that our results show previously undescribed differences in immunoreactivity among individual $\alpha, \beta, \gamma, \omega-5$ and $\omega-1.2$ gliadins. This relationship is especially evident among $\omega-5$ and $\omega-1.2$, where $\omega-5$ [2] was much more immunoreactive than $\omega-5[3]$. Differences between $\omega-1.2[1]$ and $\omega-1.2[3]$ fractions were even greater. Moreover, one $\omega-1.2$ fraction $(\omega-1.2[3]$, a weak protein band) was more immunoreactive than $\omega-5[3]$. Both $\omega$ gliadin subgroups, like other gliadins, are highly polymorphic protein groups $[23,24]$, coded by genes on different chromosomes ( $\omega-1.2$-mainly chromosome $1 \mathrm{D}$, and $\omega-5$ on chromosomes 1A and 1B). The catalogue of gliadin proteins worked out by Metakovsky [25] shows $46 \omega$-gliadin blocks, each composed on several fractions of different physicochemical properties. Blocks coded by different chromosomes may occur in all possible combinations among wheat genotypes. This emphasizes the complex structures and major differences among $\omega$-gliadins. The great diversity, as in other gliadin classes, may relate to different immunoreactive properties, and may make possible to identify the main wheat allergens responsible for pathogenesis in allergic diseases. This could also permit breeding strategies for development of hipoalergenic wheat genotypes, since knowledge about allergenicity differentiation of individual gliadin fractions may greatly facilitate selection of hybrid plants containing proteins of decreased immunoreactivity It has been proposed that $\omega-5$ gliadins (native or recombinant) may serve as the basis of an effective test to identify and diagnose food allergy in patients sensitive to gluten [26-29]. Since $\omega$-gliadins differ in immunoreactivity, choosing proper fractions for assays may significantly improve test sensitivity and specificity; isolation, purification and analysis of purified gliadin allergenicity may be important in optimizing such tests. Supposedly, use of less allergenic $\alpha$-, $\beta$ - and $\gamma$-gliadins in screening may also reveal significant information, indicating specific susceptibility to minor wheat allergens.

Our results suggest that research on immunoreactive properties cannot be restricted to gliadin groups $(\alpha, \beta, \gamma$, 
$\omega-5$ and $\omega-1.2)$, but should be extended to individual protein fractions (A-PAGE bands) within groups. Considering that many individual A-PAGE bands can be further fractionated by two-dimensional (IEF/SDS-PAGE) electrophoresis (results unpublished), one may suppose that identification of the most allergenic proteins among $\omega$-gliadins (and other gliadin groups) should be carried out on the level of individual monomeric proteins.

Results of our in vitro ELISA tests were confirmed to some extent by SPT; both methods showed an immunological response to applied protein fractions. Patient 1 with baker's asthma and WDEIA displayed a positive SPT reaction to all gliadins. However, in the patient 2 with baker's asthma but not WDEIA, only fraction ( $\omega-5$ [2]) was immunoreactive, in spite of levels of sIgE antibodies being similar (12.3 kU/L and $11.4 \mathrm{kU} / \mathrm{L}$ respectively) in both patients. In patients with skin symptoms, no positive results were observed. These reactivities suggest that $\omega-5$ gliadins, strong allergens in WDEIA, may be also immunologically active in baker's asthma. Previously, major allergens in this disease were thought to be albumins that inhibit trypsin and $\alpha$-amylase [30,31]. These observations also suggest specificity between various antigens and the type of allergic disease that they may trigger. Considering that our research was performed on only four patients, verification of these observed relationships in population studies with larger groups comprising more gluten protein susceptible persons is necessary, and will be a goal of our further research.

Based on the obtained results the following conclusions can be drawn:

- The $\alpha, \beta, \gamma, \omega-1.2$ and $\omega-5$ gliadin groups obtained from winter wheat cultivar Ostka strzelecka show significant differentiation in regard of immunoreactive properties as measured by ELISA using pooled sera of four patients with gluten intolerance.

- Immunoreactivity of both $\omega-1.2$ and $\omega-5$ gliadin subgroups is much higher than that of the other $\alpha$-, $\beta$ - and $\gamma$-gliadin groups.

- Some of individual gliadin fractions among gliadin groups and subgroups differ also significantly in regard of immunoreactivity. One of the $\omega-1.2$ gliadins was even more immunoreactive than $\omega-5$ [3] fraction what was undescribed previously.

- Purified gliadin protein fractions used as reagents for skin prick tests show differentiated immunological response in on patients who donated sera for the study, hence they may serve as the as the basis of an effective test to identify and diagnose food allergies.

\section{Acknowledgements}

This work was carried out with financial support from The Polish Ministry of Science by research grant No. N
N310 162238.

\section{REFERENCES}

[1] A. S. Tatham and P. R. Shewry, "The Conformation of Wheat Gluten Proteins. The Secondary Structure and Thermal Stabilities of the Alpha-, Beta-, Gamma- and Omega-Gliadins," Journal of Cereal Science, Vol. 3, No. 2, 1985, pp. 103-113. doi:10.1016/S0733-5210(85)80021-7

[2] C. W. Wrigley and J. A. Bietz, "Proteins and Amino Acids," In: Y. Pomeranz, Ed., Wheat Chemistry and Technology, American Association of Cereal Chemists, St. Paul, Inc., St. Paul, 1988, pp. 159-275.

[3] P. I. Payne, "Genetics of Wheat Storage Proteins and the Effect of Allelic Variation on Bread-Making Quality," Annual Review of Plant Physiology, Vol. 38, 1987, pp. 141-152. doi:10.1146/annurev.pp.38.060187.001041

[4] A. A. Sozinov and F. A. Poperelya, "Genetic Classification of Prolamins and Its Use for Plant Breeding," Annales de Technologie Agricole, Vol. 28, 1980, pp. 229-245.

[5] J. A. Bietz, "Recent Advances in the Isolation and Characterization of Cereal Proteins," Cereal Foods World, Vol. 24, 1997, pp. 199-202.

[6] J. A. Bietz, "HPLC of Cereal Endosperm Storage Proteins," In: K. M. Gooding and F. E. Regnier, eds., HPLC of Biological Macromolecules, 2nd edition, Marcel Dekker, Inc., New York, 2002, pp. 547-587.

[7] J. Waga, "Polymorphism of Gliadin and Glutenin Proteins and Their Relations to Quality of Winter Wheat (Triticum aestivum L.)," Biuletyn Instytutu Hodowli i Aklimatyzacji Roślin, Vol. 204, 1997, pp. 205-218.

[8] P. R. Shewry and A. S. Tatham, "Disulphide Bonds in Wheat Gluten Proteins," Journal of Cereal Science, Vol. 25, No. 3, 1997, pp. 207-227. doi:10.1006/jcrs.1996.0100

[9] A. S. Tatham, "The Structures of Wheat Proteins," Proceedings of a Conference: "Wheat Structure, Biochemistry and Functionality" Organised by the Royal Society of Chemistry Food Chemistry Group, Reading, 10-12 April 1995, pp. 53-62.

[10] A. S. Tatham, P. R. Shewry and P. S. Belton, "Structural Studies of Cereal Prolamins Including Wheat Gluten," Advances in Cereal Science and Technology, Vol. 10, 1990, pp. 1-78.

[11] L. Charbonnier and A. De Wolf, "Sequences N-Terminales de $3 \omega$-Gliadins dub le Capelle (T. aestivum)," Académie des Sciences, Paris, Vol. 297, 1983, pp. 181-186.

[12] M. Lehto, K. Palosuo, E. Varjonen, M. L. Majuri, U. Andersson, T. Reunala and H. Alenius, "Humoral and Cellular Responses to Gliadin in Wheat-Dependent, Exercise-Induced Anaphylaxis," Clinical \& Experimental Allergy, Vol. 33, No. 1, 2003, pp. 90-95. doi:10.1046/j.1365-2222.2003.01568.x

[13] K. Palosuo, E. Varionen, O. M. Kekki, T. Klemola, N. Kalkkinen, H. Alenius and T. Reunala, "Wheat $\omega-5$ Gli- 
adin Is a Major Allergen in Children with Immediate Allergy to Ingested Wheat," Journal of Allergy and Clinical Immunology, Vol. 108, No. 4, 2001, pp. 634-638. doi:10.1067/mai.2001.118602

[14] T. Daengsuwan, K. Palosuo, S. Phankingthongkum, N. Visitsunthorn, O. Jirapongsananuruk, H. Alenius, P. Vichyanond and T. Reunala, "IgE Antibodies to $\omega-5$ Gliadin in Children with Wheat-Induced Anaphylaxis," Allergy, Vol. 60, 2005, pp. 506-509.

[15] J. Waga and J. Zientarski, "Isolation and Purification of Individual Gliadin Proteins by Preparative Acid Polyacrylamide Gel Electrophoresis (A-PAGE) for Allergenic Research," Polish Journal of Food and Nutrition Sciences, Vol. 57, 2007, pp. 91-96.

[16] W. Bushuk and R. R. Zillman, "Wheat Cultivar Identification by Gliadin Electrophoregrams. I. Apparatus, Method and Nomenclature," Canadian Journal of Plant Science, Vol. 58, No. 2, 1978, pp. 505-515. doi:10.4141/cjps78-076

[17] S. Dreborg and A. Frew, "Position Paper: Allergen Standardization and Skin Tests," Allergy, Vol. 48, 1993, pp. 49-82. doi:10.1111/j.1398-9995.1993.tb04756.x

[18] M. Watanabe, S. Tanabe, T. Suzuki, Z. Ikezawa and S. Arai, "Primary Structure of an Allergenic Peptide Occurring in the Chymotriptic Hydrolysate of Gluten," Bioscience, Biotechnology and Biochemistry, Vol. 59, No. 8, 1995, pp. 1596-1995. doi:10.1271/bbb.59.1596

[19] S. Tanabe, S. Arai, Y. Yanagihara, H. Mita, K. Takahashuki and M. Watanabe, "A Major Wheat Allergen Has a Gln-Gln-Gln-Pro-Pro Motif Identified as an IgE-Binding Epitope," Biochemical and Biophysical Research Communications, Vol. 219, No. 2, 1996, pp. 290-293. doi:10.1006/bbrc.1996.0225

[20] M. Rumbo, F. G. Chirdo, C. A. Fossati and M. C. Anon, "Analysis of Anti-Prolamin Monoclonal Antibody Reactivity Using Prolamin Fractions Purified by Preparative Electrophoresis," Food and Agricultural Immunology, Vol. 12, No. 1, 2000, pp. 41-52. doi:10.1080/09540100099616

[21] I. Bouchez-Mahiout, J. Snegaroff, M. Tylichova, C. Pecquet, G. Branlard and M. Lauriere, "Low Molecular Weight Glutenins in Wheat Dependant, Exercise-Induced Anaphylaxis: Allegenicity and Antigenic Relationships with Omega 5-Gliadins," International Archives of Allergy and Immunology, Vol. 153, 2010, pp. 35-45. doi:10.1159/000301577

[22] M. Akagawa, T. Handoyo and T. Ishii, "Proteomic Analysis of Wheat Flour Allergens," Journal of Agricul- tural and Food Chemistry, Vol. 55, No. 17, 2007, pp. 6863-6870. doi:10.1021/jf070843a

[23] F. M. DuPont, W. H. Vensel, R. Chan and D. D. Kasarda, "Characterization of 1B-Type $\omega$-Gliadins from Triticum aestivum Cultivar Butte," Cereal Chemistry, Vol. 77, No. 5, 2000, pp. 607-614. doi:10.1094/CCHEM.2000.77.5.607

[24] C. C. Hsia and O. D. Anderson, "Isolation and Characterization of Wheat $\omega$-Gliadin Genes," Tag Theoretical and Applied Genetics, Vol. 103, No. 1, 2001, pp. 37-44. doi:10.1007/s00122-001-0552-2

[25] E. V. Metakovsky, "Gliadin Allele Identification in Common Wheat. II. Catalogue of Gliadin Alleles in Common Wheat," Journal of Genetics and Breeding, Vol. 45, 1991, pp. 325-344.

[26] H. Matsuo, K. Kohno, H. Niihara and E. Morita, "Specific IgE Determination to Epitope Peptides of $\omega-5$ Gliadin and High Molecular Weight Glutenin Subunits Is a Useful Tool for Diagnosis of Wheat-Dependent Exercise-Induced Anaphylaxis," The Journal of Immunology, Vol. 175, 2005, pp. 8116-8122.

[27] H. Matsuo, J. Dahlström, A. Tanala, K. Kohno, H. Takahashi, M. Furumura and E. Morita, "Sensitivity and Specificity of Recombinant $\omega-5$ Gliadin-Specific IgE Measurement for the Diagnosis of Wheat-Dependent Exercise-Induced Anaphylaxis," Allergy, Vol. 63, 2008, pp. 233-236

[28] H. Matsuo, K. Kohno and E. Morita, "Molecular Cloning, Recombinant Expression and IgE-binding Epitope of $\omega-5$ Gliadin, a Major Allergen in Wheat Dependent Exercise-Induced Anaphylaxis," FEBS Journal, Vol. 272, 2005, pp. 4431-4438. doi:10.1111/j.1742-4658.2005.04858.x

[29] E. Morita, H. Matsuo, S. Mihara, K. Morimoto, A. W. J. Savage and A. S. Tatham, "Fast $\omega$-Gliadin Is a Major Allergen in Wheat-Dependent Exercise-Induced Anaphylaxis," Journal of Dermatological Science, Vol. 33, 2003, pp. 99-104. doi:10.1016/S0923-1811(03)00156-7

[30] R. Sanchez-Monge, L. Gomez, D. Barber, C. Lopez-Otin, A. Armentia and G. Salcedo, "Wheat and Barley Allergens Associated with Baker's Asthma," Biochemical Journal, Vol. 281, 1992, pp. 401-405.

[31] R. Sanchez-Monge, G. Garcia-Casado, C. Lopez-Otin, A. Armentia and G. Salcedo, "Wheat Flour Peroxidase Is a Prominent Allergen Associated with Baker's Asthma," Clinical \& Experimental Allergy, Vol. 27, 1997, pp. 1130-1137. doi:10.1111/j.1365-2222.1997.tb01149.x 